\title{
Screening of patients for first time prostheses after amputation of lower limbs
}

\author{
A. Vetra ${ }^{1,2}$, K. Lacis ${ }^{1,3}$, G. Raipalis ${ }^{1,3}$, E. Legzdina ${ }^{1}$, and V. Logins ${ }^{1}$ \\ ${ }^{1}$ Rīga Stradiņš University, Riga, Latvia \\ ${ }^{2}$ National Rehabilitation center Vaivari \\ ${ }^{3}$ DDA Orthopaedics
}

\begin{abstract}
In the world's developed countries, demographic changes, such as aging societies and increased numbers of diabetes patients, have led to an increase in amputations (11), and it is believed that as society ages, the number of people who are older than 65 will face as much as two times more amputations [3].

Of great importance after the amputation of a lower limb is the selection and adaptation of prostheses that allow patients to move around, thus achieving rehabilitation goals much more quickly [5].

A 20090-study of the rehabilitation of people with lower limb amputations in Latvia found that among 183 patients, 50\% use their prostheses actively for more than 6 hours a day, 30\% did not use them at all or used them for less than 3 hours a day, and 20\% used them for 3-6 hours a day.

The study included 173 patients with lower limb amputations who were evaluated in 2012 in relation with whether primary prosthesis should be provided.

Of 173 patients who were evaluated, only 109 received a decision on primary prostheses, while in 51 cases the process was delayed for 1-3 months, in 12 cases, it was decided that prostheses would not be purposeful.

More than $25 \%$ of those who followed the recommended treatment and rehabilitation programme to prepare the amputation stump, reduced contracture and enhanced physical working abilities were declared to be appropriate for further prostheses. This indicates serious shortcomings in medical treatments during the early post-amputation period.
\end{abstract}

Throughout the world, societies are aging, and there are increasing numbers of people with chronic diseases such as chronic blood flow disorders. Peripheral arterial diseases are the most common reason for the amputation of lower limbs. Despite the development of modern medicine and technologies, amputations remains one of the leading reasons for disability in the world $[2,3]$.

In the world's developed countries, demographic changes, such as aging societies and increased numbers of diabetes patients, have led to an increase in amputations (11), and it is believed that as society ages, the number of people who are older than 65 will face as much as twice more amputations [3].

Amputation influences the patient's physical, psychological and social functions (4), as well as their role in society. Amputations do not shorten people's lives, and patients require support so that they can return to society as quickly as possible and fulfil their social roles. Good mobility is the key for functional independence and the ability to return to society for such people. 
Modern treatment, rehabilitation and prosthesis technologies mean that someone whose lower limbs have been amputated does not have to stay in bed. They can care for themselves and return to the labour market and social environment in a competitive way.

Anyone who has undergone the amputation of a limb will have an individualised rehabilitation process that is prepared in partnership with the patient. Factors that have a positive influence on rehab processes include sufficient physical activity before the amputation, a satisfactory standard of living and health situation before the amputation, positive motivation for the patient, and good contacts and communications between the patient, his family and the rehabilitation team.

Of great importance after the amputation of a lower limb is the selection and adaptation of prostheses that allow patients to move around, thus achieving rehabilitation goals much more quickly [5]. Before this, there must be early rehabilitation programmes while patients are still in hospital, ensuring prevention of complications such as bedsores, contractures and the healing of scars. Patients must learn how to treat their scars, engage in contracture prophylaxis, and shape the stump to prepare it for the prostheses. Patients must learn to use ancillary resources, stretch and strengthen their muscles, and engage in balance and mobility exercises. They must be educated about the need for such processes, and they and their relatives must be taught how to prevent complications at home. Patients must also be informed about prosthesis opportunities and prepared for them (Otto Bock Kompendium 2010).

Prostheses depend on the cause of the disease that led to the amputation, the age of the patient, and the scope of the amputation. People who suffer amputation traumas or cancers achieve better results after prostheses are adapted to their needs. 97\% of post-amputation patients can begin to walk with prostheses in three months' time [12]. Patients with peripheral bloodstream diseases and diabetes learn to walk more slowly, and it takes them longer time to achieve good results. Good results are achieved by approximately $80 \%$ of people with vascular disorders [13], and good results in terms of restoring locomotion with a prosthesis are achieved by $78 \%$ of patients who are older than 65 after transtibial amputations and $57 \%$ after transfemoral amputations.

Patients who have lost limbs have increased demands in terms of learning new skills and successful use of prostheses. Patients who use lower limb prostheses quickly grow tired because of an overload of their nervous system, not because of physical weariness. It is particularly important to motivate patients so that they understand why they must learn new skills in relation to the lower limb prosthesis. Each ancillary resource creates a new internal environment for patients, and adaptation is needed. Patients must be taught to use prostheses properly without devoting too much energy to the process. It is not recommended to allow patients to learn this themselves, because that can lead to improper use of prostheses, excessive and inadequate compensatory movements, deformation of the body or its extremities, an incorrect posture, physical overloads and pain. People who learn to use lower limb prostheses themselves need new training. This must happen under the supervision of a physiotherapist and technical orthopaedist [7, 8].

According to research, approximately 1,000 amputations of lower limbs are conducted in Latvia each year, and on average 460 lower limb prostheses are manufactured. A 2009 study of the rehabilitation of people with lower limb amputations in Latvia found that among 183 patients, 50\% use their prostheses actively for more than 6 hours a day, 30\% did not use them at all or used them for less than 3 hours a day, and $20 \%$ used them for 3-6 hours a day.

The most important thing in learning to use lower limb prostheses is the health, physical readiness and training of the patient under inpatient conditions and under the supervision of specialists. This is particularly important for patients who are receiving their first prostheses, 
as well as for patients for whom both lower limbs have been amputated. It is not enough for the state to finance prostheses and their manufacturing. There must also be financing for rehabilitation and training.

A patient who has received prostheses must immediately go through rehabilitation to learn how to walk properly, but that does not happen if the patient has had to wait for the prostheses for a long time, as much as two years before he or she receives adequate rehabilitation and training in terms of how to walk. It is also possible that the patient will end up in a rehabilitation centre, but often the patient must wait for many months before the technical assistance resource will be provided. In both cases, the patient suffers because of not receiving adequate services. The rehab team also suffers because time and resources have been wasted, because the successful supply of prostheses is not ensured. Costs that relate to care, rehabilitation and post-amputation prostheses are important.

Given the increased costs, rehabilitation programmes have problems in ensuring sufficient therapy in relation to prostheses [1]. What's more, the trend of expanding outpatient rehabilitation increases the unavailability of these services to amputees. There are transport barriers that cause problems for patients, particularly in rural and peripheral regions, in terms of visiting clinics for face-to-face meetings [16]. This means the need for more approachable and innovative approaches to improve the ability of patients with lower limb amputations to overcome these obstacles.

To improve the availability of professional rehabilitation services, many countries, including Latvia, have launched tele-rehabilitation services so as to provide professional assistance. This is one of the components of tele-medicine, making it possible to provide rehab at home with the help of information technologies [16]. This increases the scope of rehabilitation services irrespective of time, distance and opportunities, also improving the quality of life of patients who are at home. Similarly to traditional rehabilitation, telerehabilitation ensures supervision, as well as communications between the patient and the specialist. This optimises rehabilitation, particularly for patients with lower limb amputations, because a multidisciplinary team takes part in the process.

The inclusion of tele-rehabilitation in overall rehab programmes ensures ongoing care after the plan is confirmed, thus saving time and costs in terms of the repeated inpatient rehabilitation for the patient. Home-based rehab is particularly important for those who have limited access to equipment and transport. It has been shown that tele-rehabilitation can increase the effectiveness of the process of patients with lower limb amputations, involving specialists with higher qualifications in the process, reducing time and costs, and establishing a highly qualified multidisciplinary team for the rehabilitation process.

Given the aforementioned situation with amputation results in Latvia, the Technical Resource Centre (TPC) began additional evaluation of patients in 2012 - those who were sent to receive state-financed prosthesis services after lower limb amputations. There is a special evaluation protocol for such patients, including:

Therapeutic evaluation:

- An evaluation of the level of the amputation;

- A general evaluation by a physiotherapist about strength and movement in lower limbs, as well as walking with crutches or suing a wheelchair;

- Evaluation of tiny motor abilities (the nine hole test and the block test);

- Evaluation of cognitive capacities (the mini-mental scale if it seems that the capacities are limited);

- The Mobility Predictive Scale, which relates to balance, strength, the scope of moment and other evaluations of mobility potential with or without a prosthesis. 
An evaluation by the physician and a CPO evaluation:

- Evaluation of health;

- Evaluation of functional limitations;

- Establishment of rehabilitation goals and evaluation of the patient's motivation;

- Evaluation of the stump after amputation (length, contracture, reduction of soft tissue).

Conclusions about:

- The purposefulness of the prosthesis;

- The choice of the prosthesis;

- Additional medical and rehabilitation services;

- Alternative rehabilitation and technical resource services.

The therapeutic approach involves two therapists - two physiotherapists or a physiotherapist and an ergo-therapist. Evaluations take 30 minutes, during which the patient is evaluated and the protocol is prepared. The evaluation of the certified prosthetic/orthotic (CPO) aspects also takes 30 minutes, after which conclusions are drawn about:

- Whether it would be purposeful to provide prostheses, also choosing the functional level thereof;

- Whether prostheses need to be delayed for a certain period of time for medical or social reasons, also looking at additional medical or other steps to be taken;

- Whether it would not be purposeful to provide prostheses, instead recommending alternatives.

The aim of the study was to evaluate the results of the first year of selecting lower limb amputation patients for primary prostheses. The study included 173 patients with lower limb amputations who were evaluated in 2012 regarding the question whether primary prosthesis should be provided. 138 were men, 35 were women, with an average age of 61.49 years and most patients being above retirement age (65). In most cases, the amputation was needed because of chronic inervation or bloodstream disorders - arteriosclerosis (43\%), diabetes $(23 \%)$, or traumas $(22 \%)$. The level of amputation includes five cases in which the foot was amputated, 46 cases in which the amputation was below the knee, and 119 cases in which it was above the knee. In one case there was exarticulation of the knee joint, and in two cases there was exarticulation of the hip joint. Time between amputation and evaluation ranged from two weeks to 60 months, an average of 7.43 months. All patients had certification from a family doctor, surgeon, orthopaedist or other specialist to say that they were appropriate for prostheses.

Amputations were conducted for nearly one-third of the patients (57) in Rìga Eastern Hospital, which is Latvia's largest hospital, while up to 10 amputations were conducted in other hospitals. Only one-third of patients (52) were given clear instructions about what to do to ensure successful prosthesis. One-third was told to approach the family doctor, while 30 said that they received no instructions at all.

Of the patients who were observed for the first time, 80 , or slightly less than one-half, live outside of cities, 20 stated that they live at social care centres, 41 live in Rìga, and 32 live in other towns in Latvia.

53 of the patients arrived for evaluation within three months after the amputation, 52 did so between four and six months, and 27 did so a year or later after the amputation. 76 could move with crutches, the other 97 used wheelchairs. The Mobility Predictive Scale evaluation for patients using crutches was comparatively higher than for those who arrived in a wheelchair $-39.39 \%$ had good prosthesis results, while $2.42 \%$ had average results. For people in wheelchairs, the numbers were $16.97 \%$ and $16.36 \%$ respectively. The test 
was not fully conducted for $14.55 \%$ of patients, and unclear test results related to $10.30 \%$ of them.

Of the 173 patients who were evaluated, only 109 received a decision about primary prostheses, while in 51 cases the process was delayed for 1-3 months so that the patient could do various things to prepare for the process - binding the stump of the amputation to reduce soft tissue, medical exercises to reduce contracture, strengthening of physical working abilities, etc. In 12 cases, it was decided that prostheses would not be purposeful. Of the remaining 51 patients, $1 / 4$ had lower limb amputations and 3/4had above the knee amputations, and after an additional evaluation, 17 , or $10 \%$ of all patients, were told that prostheses would not be purposeful.

The discussion on additional functional evaluations on the basis of TCP, making use of the TPC AEANP, first of all involved the reason for primary prostheses in terms of the declaration by doctors that this would not be in line with the patient's actual functioning abilities, which would make it possible to use prostheses as an evaluation of rehabilitation technology mobility, because prostheses were rejected for $15 \%$ of all of the patients. Sadly, it is difficult to talk about the reasons why these conclusions were inappropriate - a mistake by the doctor, insufficient experience with prostheses, the lack of a critical approach by the patient, or insistence on the patient's rights, as strengthened by universally known limitations about environmental physical accessibility. This, in turn, means that amputation is very unpromising when it comes to future functions. The fact that patients who arrived for their evaluation on crutches were more appropriate candidates than those who use a wheelchair is based on scholarly literature about this subject. More than $25 \%$ of those who followed the recommended treatment and rehabilitation programme to prepare the amputation stump, reduce contracture and enhance physical working abilities were declared to be appropriate for further prostheses. This indicates serious shortcomings in medical treatments during the early post-amputation period.

It is also true that it was difficult to evaluate links between the residence of patients and the availability of treatment and rehab services, but the large number of amputees outside Rìga means that tele-rehabilitation services should be organised for them when they leave the hospital, because other realistic offers on increasing the availability of such services do not exist at this time.

Further research is needed about TPC AEANP as a high-quality protocol, and that also includes analysis of late results. Right now, however, it can be recommended that the evaluation of upper limbs should be supplemented with an evaluation of the scope of movement and the objectives of strength so as to evaluate the potential for using crutches and to propose an objective balance evaluation in the protocol. It is also necessary to include self-evaluation of the patient's environment in the evaluation of functionality. Further research is also needed to evaluate tiny motor approaches toward forecasting the results of prostheses.

\section{Conclusions}

1. The TPC-launched additional functioning of amputees on the basis of TPC/AEANP is purposeful and should be further developed with additional research and improvements to the protocol and the self-evaluation process;

2. Recommendations that amputees receive when they leave hospital are often inadequate for adequate prosthetic processes;

3. More research is needed on the use of tele-rehabilitation for the rehabilitation of amputation patients. 


\section{References}

[1] Buckley JG, O'Driscoll D, Bennett SJ. Postural sway and active balance performance in highly active lower-limb amputees. Am J Phys Med Rehabil. 2002 Jan; 81(1):13-20.

[2] Cason J, Cohn ER. Telepractice: An overview and best practices. ASHA Perpectives.2014; 23(1):4-17.

[3] Dade D. Fletcher, Karen L. Andrews, Matthew A. Butters, Steven J. Jacobsen, Charles M. Rowland, John W. Hallett Jr Rehabilitation of the geriatric vascular amputee patient: A population-based studyArchives of Physical Medicine and Rehabilitation, 82(6), June 2001, Pages 776-779

[4] M. Davidson, J. L. Keating A Comparison of Five Low Back Disability Questionnaires: Reliability and ResponsivenessPhysical Therapy, January 2002, 82(1) 8-24

[5] Ekeland A.G., Bowes A, Flottorp S Effectiveness of telemedicine: a systematic review of reviews. Int J Med Inform. 2010 Nov; 79(11): 736-71.

[6] Hoenig H, Sanford J.A. Butterfield T, Griffiths PC, Richardson P, Hargraves K

[7] Development of a teletechnology protocol for in-home rehabilitation. J Rehabil Res Dev. 2006 Mar-Apr; 43(2): 287-98.

[8] Kuipers P, Foster M, Smith S, Fleming J Using ICF-Environment factors to enhance the continuum of outpatient ABI rehabilitation: an exploratory study. Disabil Rehabil. 2009; 31(2): 144-51.

[9] Lācis K. Cilvēku ar apakšèjās ekstremitātes amputācijām rehabilitācija.Maǵistra darbs.2009, lpp.78.

[10] Robert A. Bedotto, PT, CPO, CPI Biomechanical Assessment and Treatment in Lower Extremity Prosthetics and Orthotics: A Clinical Perspective, Phys Med Rehabil Clin N Am 17 (2006) 203-243.

[11] Marzen-Groller K, Bartman K. Building a successful support group for postamputation patients. J Vasc Nurs. 2005 Jun; 23(2): 42-5.

[12] J. MacKenzie, M. J. Bosse, R. C. Castillo, D. G. Functional Outcomes Following Trauma-Related Lower-Extremity Amputation J Bone Joint Surg Am, 2004 Aug; 86(8): 1636-1645.

[13] Miller W.C., Deathe A.B. A prospective study examining balance confidence among individuals with lower limb amputation. Disabil Rehabil. 2004 Jul-Aug; 26(14-15): 875-881.

[14] Miller WC, Deathe AB, Speechley M. Psychometric properties of the Activitiesspecific Balance Confidence Scale among individuals with a lower-limb amputation. Arch Phys Med Rehabil. 2003 May; 84(5): 656-661.

[15] Miller W.C., Speechley M, Deathe B. The prevalence and risk factors of falling and fear of falling among lower extremity amputees. Arch Phys Med Rehabil. 2001 Aug; 82(8): 1031-1037.

[16] Tousignant, M., Boissy, P., Moffet, H., Corriveau, H., Cabana, F., et al. Patients' Satisfaction of Healthcare Services and Perception with In-home Telerehabilitation and Physiotherapists' Satisfaction Toward Technology for Post-knee Arthroplasty: An Embedded Study in a Randomized Trial. 2011, Telemedicine Journal and e-Health $17.5,376-82$. 